\title{
NEGATIVE CHARGED PION PRODUCTION ON A DEUTERON BY QUASI-REAL PHOTONS
}

\author{
Dmitriev V.F. ${ }^{1}$, Dusaev R.R. ${ }^{2}$, Gauzstein V.V. ${ }^{2}$, Loginov A.Yu. ${ }^{2}$, \\ Nikolenko D.M. ${ }^{1}$, Rachek I.A. ${ }^{1}$, Sidorov A.A. ${ }^{2}$, Stibunov V.N. ${ }^{2}$, \\ Shestakov Yu.V. ${ }^{1}$, Toporkov D.K. ${ }^{1}$, Zevakov S.A. ${ }^{1}$ \\ ${ }^{l}$ G.I.Budker Institute of Nuclear Physics, Novosibirsk, Russia; \\ ${ }^{2}$ National Research Tomsk Polytechnic University, Russia \\ E-mail: stib@tpu.ru
}

Experimental differential cross sections of photoproduction of negative pions on a deuteron have been obtained. A special feature of the experiment reported here is the detection of both proton in the final state of the reaction, and with large values of the momenta. The experiment was performed on an internal target of the VEPP-3 electron storage ring. In the approximation of zero scattering angles of the electrons, we investigate the reaction of production of $\pi^{-}$mesons by quasi-real photons. Coincidence detection of two protons with large momenta suppresses the mechanism of quasi-free photoproduction by increasing the relative contribution of more complex reaction mechanisms. The kinematics of pion photoproduction on a deuteron is fully reconstructed from the measured energies and proton emission angles. The calculated photon energies lie in the range $290-900 \mathrm{MeV}$. The theoretical model [1] lying at the basis of the generation of events takes into account the contribution of the diagrams of the impulse approximation and the diagram of pion-nucleon and nucleonnucleon rescattering. The elementary amplitude of photoproduction of a pion on a nucleon in the model takes into account the Born contributions, the contributions of the six nucleon resonances, and the exchange contributions of the vector mesons $\rho$ and $\omega$. The agreement of the experimental data with the theoretical predictions arrived at within the traditional framework of the impulse approximation with $\pi N$ and $N N$ rescattering speaks of the fact that the contributions of all the most important resonances from second resonance region are taken into account in the elementary amplitude of pion photoproduction on a nucleon.

This work was supported in part by the RFBR under grants № 08-02-00560-a and № 12-02-33140.

1. А.Ю.Логинов, А.А.Сидоров, В.Н.Стибунов // ЯФ. 2000. Т.63. С.459. 